\title{
Conceptos de aceptación y adaptación en discapacidades mayores
}

\author{
The concepts of acceptance and adaptation in major disabilities
}

\section{Sr. Editor:}

En la literatura científica del campo de la psicología de la rehabilitación en discapacidades mayores, es común el uso de los términos adaptación y aceptación sin hacer la distinción específica entre ambos.

La adaptación se entiende como "el estado en el que el sujeto establece una relación de equilibrio y carente de conflictos con su ambiente social" (1). Como concepto biológico, el Diccionario de la Lengua Española la define como "Acomodarse a las condiciones de su entorno" (2).

En relación a la aceptación, la Real Academia Española define aceptar como: "Recibir voluntariamente y sin oposición lo que se da, se ofrece y encarga" y." aprobar, dar por bueno, acceder a algo y asumir resignadamente un sacrificio, molestia o privación" (3).

Li y Moore (4) y Nicholls et al (5), señalan que la aceptación de la discapacidad está directamente relacionada con la aceptación de la pérdida, aunada a un proceso de revalorización de capacidades y actitudes, al grado de reconocerlas y actualizarlas como recursos y herramientas distintas a las relacionadas con la discapacidad. Con esto, se resta importancia a los aspectos de la apariencia y limitaciones físicas y, en su lugar, se enfatizan los propios activos, habilidades y fortalezas.

Diversos estudios determinan algunas variables, como la autoestima y el apoyo social, relacionadas con la aceptación de la discapacidad, el cual también se ha identificado como un elemento importante para el ajuste a la discapacidad. Sin embargo, pocos son los estudios que la relacionan con otras categorías como características demográficas, condiciones de discapacidad y otros factores psicosociales (4-6).

En la práctica clínica, se considera que dentro del proceso de rehabilitación, el paciente debe lograr en un primer momento un grado mínimo de aceptación de su estado y condición actual antes de adaptarse para instalar las bases y continuidad de la rehabilitación integral.

Los pacientes con discapacidades mayores (amputación o lesión medular), al inicio del tratamiento integral, impresionan dispuestos a su rehabilitación, gracias al medio físico que la institución les brinda (ausencia de barreras arquitectónicas, atención personalizada, etc.), lo que les devuelve la confianza en sí mismos durante su estancia hospitalaria.

Cuando este espacio cambia y el paciente sale de la institución a enfrentar el medio y su realidad - que son diferentes en lo social, infraestructural y económico institucional -, la adaptación puede fracasar si antes no se ha logrado y conseguido un mínimo de aceptación emocional. Se aprecia que en la mayoría de los casos se requiere más trabajo psicológico; lamentablemente, los tiempos de tratamiento integral no son acordes con los que requiere el psicólogo para manejar y trabajar mejor la aceptación.

La mayoría de los estudios respecto a estos constructos no hacen distinción entre aceptación y adaptación (4), mientras que nosotros, a partir de nuestra experiencia y en concordancia con unos pocos autores (5), hacemos una diferenciación entre los términos aceptación (como un proceso personal, emocional, e individual) y adaptación (entendida como un proceso de ajuste social al medio). 
Por lo mencionado, se sugiere tener en cuenta los conceptos adaptación y aceptación que permitan tener un mejor abordaje y manejo emocional en pacientes con discapacidades mayores, así como contar con instrumentos que permitan medir dichos procesos

\section{Pedro Enrique Reátegui-Vargas ${ }^{1 a}$,} Verónica D. Neciosup-Tomé ${ }^{\text {1a }}$

\section{REFERENCIAS BIBLIOGRÁFICAS}

1. Consuegra N. Diccionario de psicología. 2da ed. Bogotá: Ecoe; 2010. p. 376.

2. Real Academia Española. Adaptar. En: Real Academia Española. Diccionario de la lengua española. Madrid: Real Academia Española; 2017. (Fecha de acceso: 01 de octubre del 2018) Disponible en: http://dle.rae.es/?id=0hMBUwM
3. Real Academia Española. Aceptar. En: Real Academia Española. Diccionario de la lengua española. Madrid: Real Academia Española; 2017. (Fecha de acceso: 01 de octubre del 2018) Disponible en: http://dle.rae.es/?id=0NYmQ7a

4. Li L, Moore D. Acceptance of disability and its correlates. J Soc Psychol. 1998; 138(1):13-25.

5. Nicholls E, Lehan T, Plaza SLO, et al. Factors influencing acceptance of disability in individuals with spinal cord injury in Neiva, Colombia, South America. Disabil Rehabil. 2012; 34(13):1082-1088.

6. Attawong T, Kovindha A. The influencing factors of acceptance of disablility in spinal cord injured patients. Nepal J Neurosci. 2005; 2(1):67-70.

Recibido: 05/10/2018 